\title{
ANALYSIS OF THE FK5 SYSTEM IN THE EQUATORIAL ZONE
}

\author{
YU.B. KOLESNIK \\ Institute of Astronomy of the Russian Academy of Sciences \\ 48 Piatnitskaya St. \\ 109017 Moscow \\ Russia
}

\begin{abstract}
. 15 catalogues produced in the eighties and 12 catalogues of the sixties-seventies have been used to assess the consistency of the FK5 system with observations in the declination zone from $-30^{\circ}$ to $30^{\circ}$. Positions of the FK4-based catalogues have been transformed at the equinox and equator J2000.0.Classical $\delta$-dependent and $\alpha$-dependent systematic differences (Cat-FK5) have been formed for individual instrumental systems of the catalogues by a method close to the classical Numerical Method. The weighted mean instrumental systems for the two subsets of catalogues centered at the epochs 1970 (MIS 60-70) and 1987 (MIS 80) and for all types of systematic differences have been constructed. The mean errors of the total systematic differences in $\alpha$ and $\delta$ have been estimated as 14 mas and 21 mas, respectively, for the catalogues of the 6070 ies, and 10 mas in both $\alpha$ and $\delta$ for the catalogues of the 80ies.
\end{abstract}

It has been found that the mutual consistency of individual instrumental systems of catalogues of the 80ies with respect to $\delta$-dependent systematic differences is superior by the factor 1.5 comparing with the catalogues of 60-70ies, while the consistency of both catalogue selections with respect to $\alpha$-dependent systematic differences is comparable. Random accuracy of the FK5 positions and proper motions at the epochs under analysis has been assessed as close to expected from the formal considerations. Actual systematic discrepancies of the FK5 with observations at the respective epochs have been detected. For systematic differences $\Delta \alpha \delta \cos \delta$ and $\Delta \delta_{\delta}$, the absolute deviations of the MIS 80 are, in general, within 40 mas, those of the MIS 60-70 are within 30 mas. For systematic differences $\Delta \alpha_{\alpha} \cos \delta$ and $\Delta \delta_{\alpha}$, the absolute deviations reach 3040 mas for both MIS. For total systematic differences, local deformations of the FK5 system in the equatorial zone in both right ascension and declination has been found exceeding expected ones from the formal errors of the FK5 system by a factor about 1.5 for the MIS 60-70, and by a factor about 2 for the MIS 80. Consistency in area distribution between both MIS for the total systematic differences $\Delta \alpha \cos \delta$ has been detected. Quick degradation of the FK5 system with time due to optimistic estimation of the errors of its proper motion system is supposed to be one of the main causes of its discrepancies with observations. The results in declination are recognized to be less reliable due to larger inconsistency of the individual instrumental systems.

Before the next space astrometric mission will be realized, ground-based observations will continue to be the only available check of an external systematic accuracy of the HIPPARCOS catalogue. Evidently, random and, possibly, systematic accuracy of each individual catalogue observed from the Earth surface would be inferior to that of the HIPPARCOS catalogue. Taken as an ensemble, however, a certain selection of catalogues might give a rather definite idea about the actual distortions of the HIPPARCOS system. This study shows to which level of accuracy such ensembles of different selections of catalogues might check the HIPPARCOS system in the equatorial zone. The analysis of the FK5 gives also an idea about levels of random and systematic discrepancies which may be expected in the equatorial zone when the HIPPARCOS catalogue will be compared with the FK5 at different epochs. 